\title{
Comportement dynamique d'une turbine Kaplan lors d'un arrêt d'urgence - Application au cas de Fessenheim
}

\author{
Dynamic behaviour of a Kaplan turbine in an emergency stop situation - \\ Case study : Fessenheim
}

par D. Courteau, E. Presutti

EDF-DTG

Following rupture of a blade of the Fessenheim Kaplan turbine, investigations have been carried out to identify the probable causes, and protective solutions to be implemented. This research has led to modification of the blades and wicket gate movement laws during the release phase, in order to limit the risks linked to: exceeding the maximum speed and pressure allowed, lifting the group, appearance of impacts on the runner and draft tube wall.

\section{I $\square$ COMPORTEMENT D'UNE TURBINE KAPLAN EN PHASE D'ARRÊT D'URGENCE}

Le traitement des incidents en exploitation des groupes hydrauliques, après ouverture du disjoncteur, conduit à des positions de retour du groupe en situation de sécurité après déclenchement suivant deux principaux types de traitement :

- l'un sur défaut de type "Extérieur" (DE) conduisant à un retour en position de marche à vide à la vitesse de synchronisme par une diminution de la charge hydraulique réalisée par la fermeture rapide des pales et du vannage. Cette séquence conduit temporairement à une survitesse du groupe. Les pales poursuivant leur fermeture, l'automatisme donne l'ordre d'ouverture du vannage, afin de réaliser le réglage en vitesse du groupe en vue de la reprise de la charge dès la disparition du défaut,

- l'autre sur défaut de type "Bloqué" (DB) conduisant à l'arrêt complet du groupe par une fermeture rapide et complète du vannage, les pales pouvant être envoyées à l'ouverture ou à la fermeture suivant les installations. La première solution favorise le ralentissement du groupe par augmentation du couple de barbotage et limite le soulèvement par la réduction de la portance sur les pales. La seconde solution permet de limiter le débit d'emballement du groupe en cas d'impossibilité de fermeture du vannage associée à une manœuvrabilité insuffisante ou nulle de la vanne de garde. Dans le cas de Fessenheim, cette dernière solution est utilisée avec une phase de réouverture des pales dès l'atteinte d'un seuil de vitesse voisin de $90 \%$ du synchronisme. On observe, comme pour le cas précédent, un passage temporaire du groupe en survitesse dans les premiers instants.

Les durées de pleine fermeture du vannage sont en général comprises entre 6 et 15 secondes avec des lois de manœuvre de type simple ou double pente, celles des pales variant d'une dizaine à plusieurs dizaines de secondes avec des lois à simple pente.

Le comportement des turbines Kaplan sur déclenchement est directement conditionné par :

- les caractéristiques de la turbine (Ns, Q, H, N),

- la configuration du circuit (inertie de l'adduction, niveaux amont et aval),

- le poids des parties tournantes,

- les performances des organes de garde et du système de régulation.

La définition de lois de manœuvres adaptées doit intégrer les contraintes directement liées au dimensionnement de l'installation :

- le respect des valeurs admissibles de la pression dans les conduits d'amenée et de fuite, de la vitesse de rotation maximale du groupe,

- le non soulèvement du groupe,

- l'absence de chocs sur les pales et la paroi de l'aspirateur.

\section{II — DESCRIPTION DES PHÉNOMÈNES [2]}

\subsection{Soulèvement du groupe}

Au moment du déclenchement, le triangle des vitesses à l'entrée des pales se déforme. La fermeture du vannage provoque une coupure du débit, parallèlement la vitesse d'entraînement augmente fortement avec la vitesse de rotation de la roue. La vitesse relative a alors tendance à s'opposer à la vitesse d'entraînement provoquant une inversion des champs de pression sur les pales. Le couple devient résis- 
tant, le groupe ralentit et la poussée hydraulique s'inverse. La charge sur le pivot diminue et le groupe a alors tendance au soulèvement.

\subsection{Chocs [1]}

On peut décrire qualitativement le phénomène de la façon suivante : l'écoulement dans la turbine pendant un déclenchement n'est pas optimal, la forte déconjugaison des pales et du vannage provoque des décollements et l'apparition de la cavitation en sortie de pales. L'écoulement devient tourbillonnaire et peut entraîner la création d'une torche au centre de la roue et dans l'aspirateur. La diminution rapide du débit liée à la fermeture du vannage crée une dépression axiale, le volume de la torche s'étend dans la partie centrale sous la roue. Ce phénomène peut être assimilé à l'effet "pompe" de la roue en phase de ralentissement qui est supérieur à l'effet "moteur" de la chute. En effet, la roue a alors un rôle d'aspiration lié à son inertie alors que le vannage est pratiquement complètement fermé. Cette situation peut conduire, lors d'une phase de ralentissement rapide, à l'amplification du freinage du groupe et à l'apparition de niveaux élevés de pression en paroi sous la roue et dans l'aspirateur. Dans le cas le plus défavorable, la non conservation du débit entre le vannage et les pales peut entraîner une rupture de l'écoulement par passage au vide. Le mouvement de reflux au moment du collapse (appelé couramment "marteau d'eau") crée une remontée brutale de la pression locale qui peut conduire à la destruction de matériels tels que les pales, la porte d'aspirateur ou la pivoterie.

\section{III — CAS DE FESSENHEIM ([3] ET [4])}

\subsection{Présentation de la centrale}

La Centrale de Fessenheim est la troisième centrale située sur le Grand Canal d'Alsace. Elle est équipée de 4 turbines Kaplan SFAC de 45 MW de puissance unitaire turbinant un débit de $350 \mathrm{~m}^{3} / \mathrm{s}$ sous $15,1 \mathrm{~m}$ de chute nette et d'un poids total des parties tournantes de 540 tonnes. Chaque roue comporte 4 pales de diamètre extérieur $6,67 \mathrm{~m}$ pesant 9 tonnes chacune. Une particularité est que seul le groupe 2 est équipé de 2 jeux de soupapes d'aération en série sur le fond central, l'une étant directement pilotée par le niveau de dépression sur le fond central, l'autre étant directement reliée à la commande de fermeture du vannage. Un incident causé par un défaut biphasé sur le transformateur du groupe 1 a conduit au déclenchement de la machine sur défaut de type "DB". Les premières investigations ont révélé que les pales étaient en position ouverte, que l'une d'elles était sortie de son logement et avait "brouté" sur le manteau de roue. Par la suite, le démontage complet de la turbine a révélé la rupture de type fragile de la dite pale au droit du tourillon. L'analyse métallurgique des différentes pales a par ailleurs révélé des caractéristiques mécaniques globalement basses compte tenu du matériau avec notamment une résilience très faible de la pale cassée. Une première campagne d'essai menée sur le groupe 2 a mis en évidence des dysfonctionnements lors des phases d'arrêt. Les analyses réalisées ont conduit à orienter les investigations sur une cause d'origine hydraulique, des phénomènes d'ordre purement mécanique ou électrodynamique n'étant pas pertinents.

\section{- 3.2 Démarche retenue}

La démarche a été conduite en deux temps afin d'explorer tout d'abord les solutions mettant en œuvre des lois à simple pente qui permettaient de minimiser les modifications sur les circuits de commande des pales et du vannage. Dans un second temps, compte tenu de l'inefficacité de l'étape précédente, les solutions de type lois à double pente ont été explorées. Pour chaque étape l'instrumentation et les lois de manœuvre testées ont été définies dans une première approche au travers d'un modèle numérique, puis ajustées par des essais in situ. Elles ont été menées pour les couples et types de lois de fermeture suivants :

\section{Lois simple pente :}

Couple $I$ (valeurs initiales) : vannage en 7,2 secondes, pales en 10 secondes (annexe 2)

Couple 2 : vannage en 8 secondes, pales en 20 secondes Couple 3 : vannage en 9 secondes, pales en 40 secondes Couple 4 : vannage en 10 secondes, pales en 60 secondes

Lois double pente : Pales en 40 secondes

Couple 5 : pleine fermeture vannage :

lère pente en 6 secondes (annexe 4)

2ème pente en 30 secondes

Point de cassure à $33 \%$ d'ouverture.

Couple 6 : pleine fermeture vannage :

lère pente en 6 secondes (annexe 5)

2ème pente en 30 secondes

Point de cassure à $42 \%$ d'ouverture.

Les paramètres suivants ont été enregistrés (voir ann. 1) : - les pressions sur la bâche, le fond turbine, le manteau de roue et l'aspirateur,

- les déplacements des pales, du vannage et des soupapes d'aération,

- la vitesse de rotation,

- la puissance électrique et les niveaux amont et aval,

- le déplacement axial de l'arbre,

- pour certains essais, la poussée axiale et le couple sur l'arbre ainsi que des mesures de contraintes sur la porte aspirateur.

\section{- 3.3 Analyse des phases d'arrêt du groupe}

\subsubsection{Etat initial}

Les enregistrements (voir annexe 2 figure 1) indiquent un niveau de vitesse maximale instantanée voisin de la limite admissible pour une puissance inférieure à la valeur nominale. On observe par ailleurs que la pression sous la roue tombe au vide, les fluctuations de pression liées au sillage des pales disparaissent, puis de fortes fluctuations de pression apparaissent atteignant jusqu'à 4,4 bars pour 0,2 bar en régime permanent. Parallèlement, la pression aspirateur s'abaisse pour entrer dans une zone à fortes fluctuations avec un maximum atteignant 1,9 bar pour 0,5 bar en régime permanent, la pression à l'amont réagit de même révélant la propagation d'ondes vraisemblablement liées à des implosions de poches de vide. De même, la pression sur le fond central s'abaisse fortement pour atteindre le vide, dans le même temps les soupapes s'ouvrent, la poussée axiale et la vitesse présentent alors des discontinuités. Ces observations traduisent la présence de chocs sous la roue liés à une phase de ralentissement brutal du groupe. 


\subsubsection{Evolution des différents paramètres}

Vitesse de rotation (voir fig. 1)

Pour les lois de manœuvre à simple pente (couples 1, 2, 3 et 4), l'augmentation des temps de fermeture du vannage conduit à réduire les vitesses de fermeture des pales afin de respecter la limite admissible. On observe sur ce point une légère augmentation de la survitesse. La mise en œuvre d'une loi à double pente permet quant à elle de réduire de façon significative le niveau de survitesse, le déplacement du point de cassure est par contre sans influence (couples 5 et 6 ).

Pressions sous la roue (voir fig. 2)

L'allongement des temps de fermeture pour les lois à simple pente conduit à une amplification des niveaux de pression sur le manteau de roue aux fortes charges. Ces observations peuvent s'expliquer par l'apparition de chocs sous la roue et sont certainement dues à une forte déconjugaison du groupe ainsi qu'à une faible contre-pression aval (de l'ordre de 0,1 bar - voir annexe 3). La mise en œuvre d'une loi de fermeture à deux pentes provoque une réduction significative de ces niveaux de pression.

\section{Evolution du soulèvement (voir fig. 3 )}

L'impact d'une loi à double pente ainsi que celui des soupapes d'aération sont clairement identifiés pour limiter le soulèvement.

Pour les lois à simple pente, la marge par rapport au soulèvement c'est-à-dire au déchargement complet de la pivoterie est très faible voire nulle, elle n'est par ailleurs pas augmentée par l'allongement des temps de fermeture des pales et du vannage. La mise en œuvre de lois à double pente permet de réduire de façon significative le soulèvement. L'utilité des soupapes d'aération est clairement confirmée par son effet de limitation du soulèvement.

Evolution du couple de freinage (voir fig. 4)

Pour une loi simple pente, une image du couple sur l'arbre

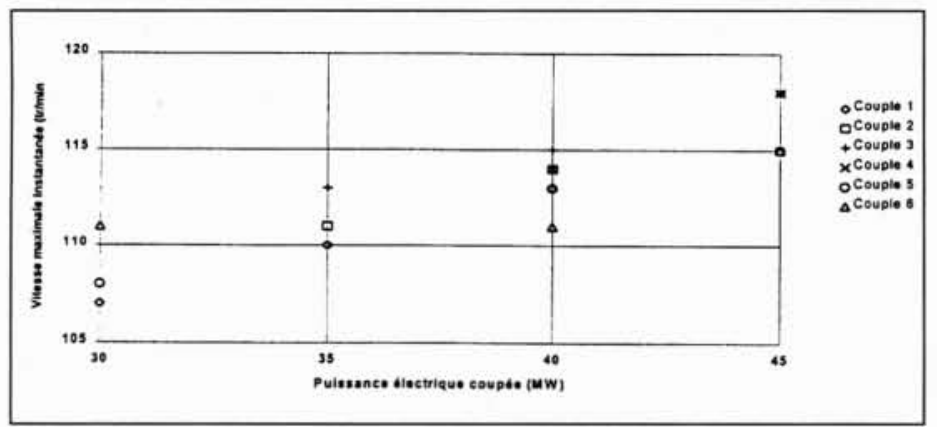

1. Evolution de la vitesse maximale instantanée sur déclenchement de type DB.

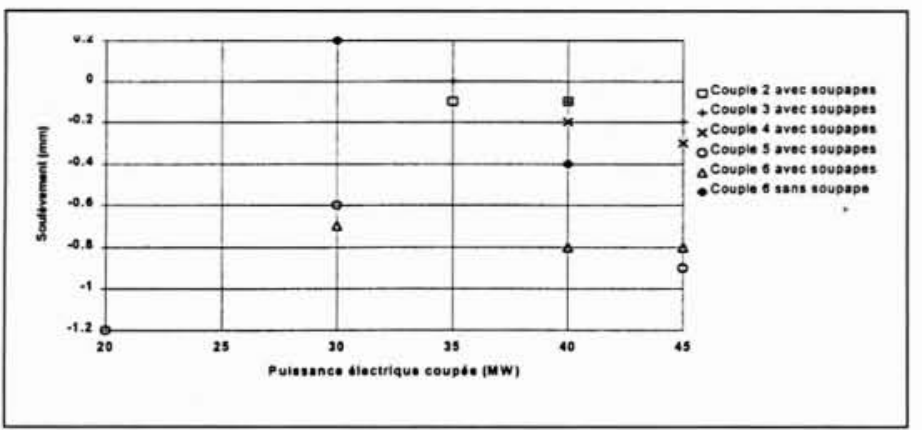

3. Evolution du soulèvement sur déclenchement de type DB. à partir de la dérivation du signal de vitesse a mis en évidence un couple de freinage de l'ordre de grandeur du couple nominal qui constitue une valeur élevée d'autant que le ralentissement est relativement brutal en atteignant environ $50 \%$ du couple nominal (voir annexe 2 fig. 2). Les relevés du couple sur l'arbre indiquent une réduction sensible du couple de freinage liée au passage à une loi à double pente pour des fonctionnements avec des soupapes d'aération en service. Le décalage du point de cassure vers les ouvertures plus fortes conduit au même effet. Dans le même temps, la courbe de ralentissement du groupe tend vers une allure monotone décroissante. Par ailleurs, l'influence du système d'aération ne joue pas un rôle significatif sur la réduction du couple de freinage.

\section{IV — RÉSULTATS [4]}

\section{- 4.1 Influence des lois simple pente}

L'augmentation des temps de fermeture des pales et du vannage permet de réduire les variations brutales sur les signaux de poussée axiale et de déplacement mais conduit à une augmentation sensible des fluctuations de pression sous la roue. Ce comportement peut être attribué à une plus forte déconjugaison de la turbine dans la phase de ralentissement. De plus les marges par rapport au risque de soulèvement et de dépassement de la survitesse admissible restent faibles. L'amélioration des conditions transitoires d'arrêt sur déclenchement impose l'adaptation d'une loi de fermeture à deux pentes.

\subsection{Influence des lois à double pente}

L'optimisation de la loi de fermeture du vannage à double pente a mis en évidence les points suivants :

- la première pente a pour effet de rabattre la survitesse et par conséquent le surdébit tout en respectant la limite admissible en surpression dans le conduit amont,

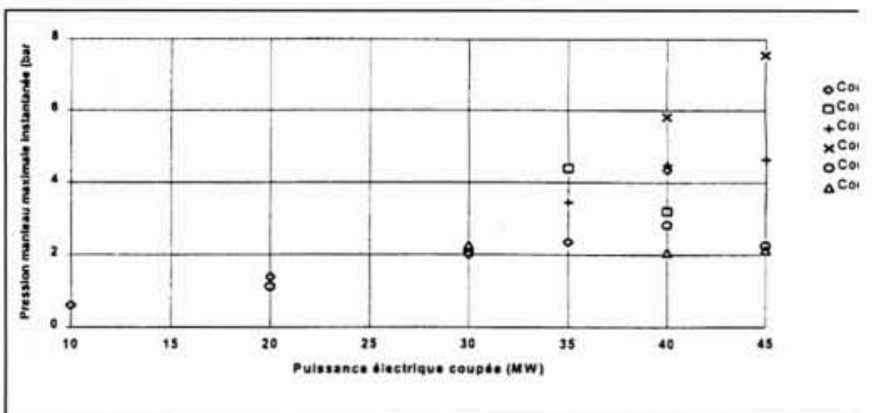

2. Evolution de la pression manteau maximale instantanée : déclenchement de type DB.

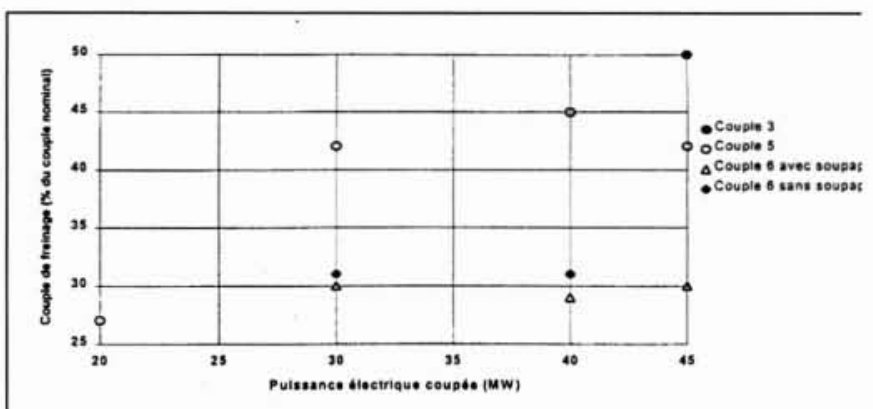

4. Evolution du couple de freinage sur déclenchement de type $\mathrm{L}$ 
- la première et la seconde pente, la position du point de cassure et la loi de fermeture des pales permettent de limiter le soulèvement par la réduction de la vitesse de rotation en fin de fermeture du vannage,

- la seconde pente et la position du point de cassure permettent de réduire le couple de freinage et d'améliorer la phase de ralentissement en limitant les risques de chocs sous la roue.

\section{- 4.3 Influence des soupapes d'aération}

On note que l'utilité des soupapes d'aération est clairement confirmée par son effet de limitation du soulèvement. En effet, l'introduction d'air autour du moyeu dénoie partiellement les pales côté tourillon, ce qui contribue à diminuer la réaction hydraulique de barbotage donc le soulèvement du groupe.

\section{$V \square$ CONCLUSION}

Le comportement du groupe de Fessenheim est rendu acceptable avec une loi de manœuvre du vannage à double pente en présence de soupapes d'aération sur le fond central.

L'optimisation des lois de fermeture des pales et du vannage sur une turbine Kaplan lors de déclenchement est nécessaire dans les cas d'équipement, de renouvellement et de rénovation. Elle doit permettre de résoudre les contraintes suivantes :
- la survitesse et la surpression maximales admissibles,

- le soulèvement du groupe,

- les risques de chocs sur la roue et la paroi de l'aspirateur.

Les moyens actuels de modélisation numérique peuvent permettre un premier niveau de définition des lois de fermeture qui reste cependant incomplet. En effet, la modélisation des phénomènes physiques n'est pas encore complètement adaptée pour qualifier les phénomènes de chocs et de soulèvement, il est donc nécessaire, d'une part, d'effectuer un recalage précis sur la base de résultats expérimentaux et plus particulièrement sur le paramètre vitesse et, d'autre part, de valider par des mesures in situ les solutions préconisées.

\section{Références}

[1]. Centrale de Bollène - Problème des chocs consécutifs à des déclenchements - Bilans des essais du G6. EDF/DTG - M. SIMONNOT - décembre 1983

[2]. Contribution à l'étude du comportement des turbines Kaplan en régime transitoire. EDF/DTG - D. LEFEVRE - juillet 1984

[3]. Centrale de Fessenheim - Groupe 2 - Comportement du groupe sur déclenchement - Essais d'Avril et Mai 1996. EDF/DTG ( D4134/RAP/97 013) - E. PRESUTTI - Avril 1997

[4]. Centrale de Fessenheim - Groupe 2 - Mise en place d'une loi de fermeture du vannage à deux pentes - Essais d'Octobre 1996. EDF/DTG (D4134/RAP/97-015) - E. PRESUTTI - Avril 1997

\section{ANNEXE 1 : FESSENHEIM GROUPE 2 : SCHÉMA D'IMPLANTATION DES POINTS DE MESURES}

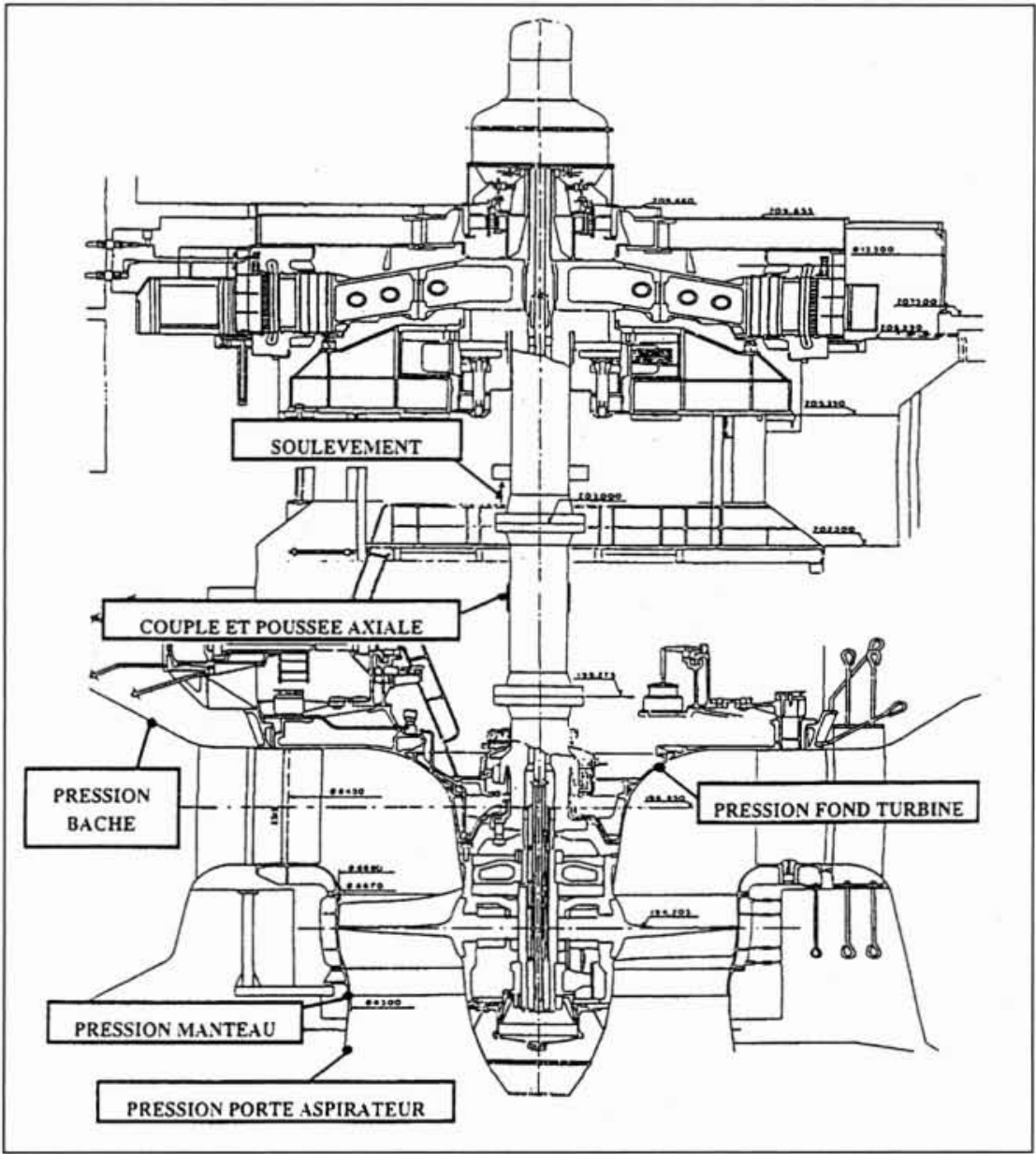


ANNEXE 2
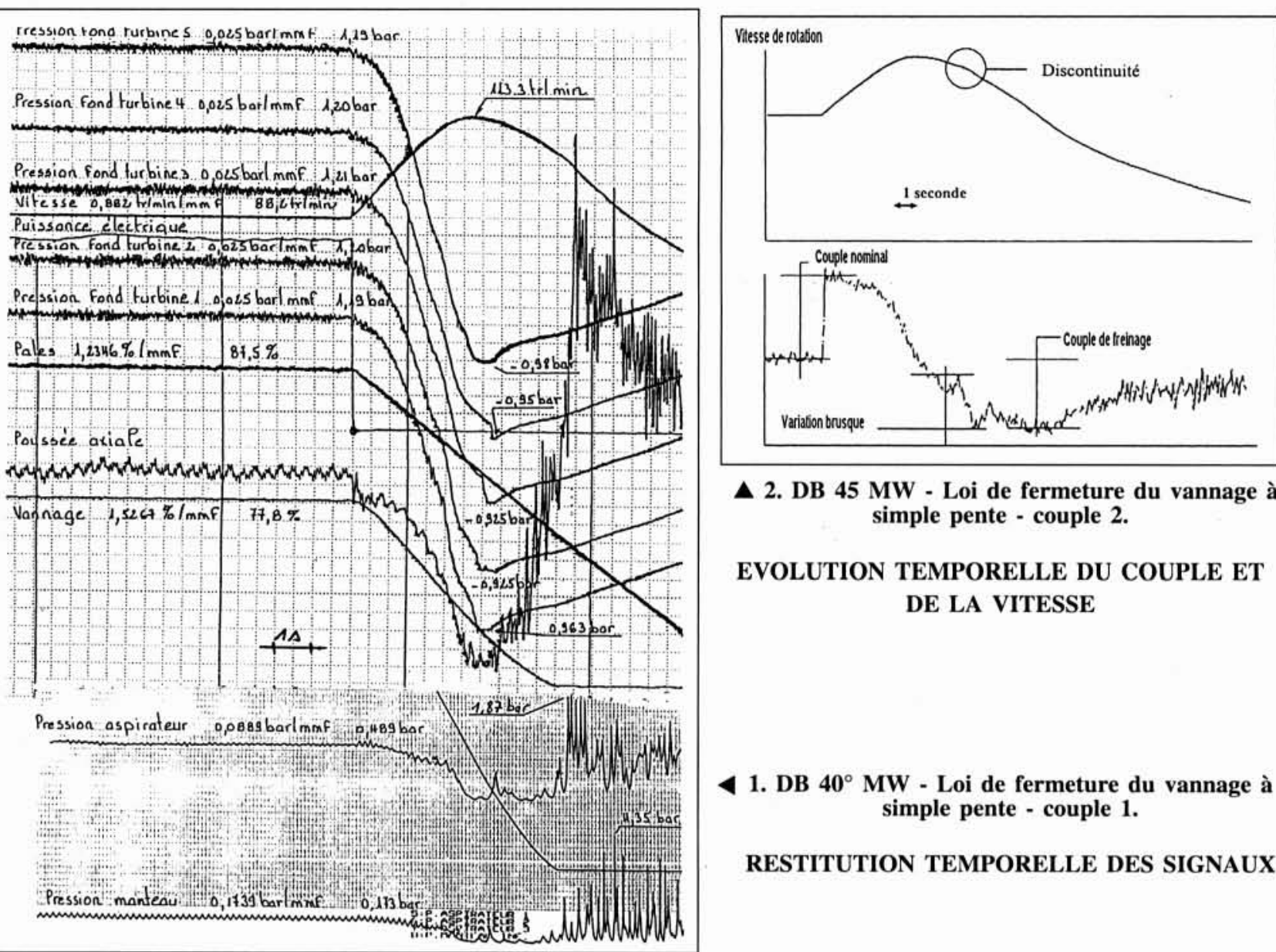

$\Delta$ 2. DB 45 MW - Loi de fermeture du vannage à simple pente - couple 2 .

EVOLUTION TEMPORELLE DU COUPLE ET DE LA VITESSE

ANNEXE 3 : RESTITUTION TEMPORELLE D'UN ESSAI DE DÉCLENCHEMENT TYPE DB À 45 MW LOI DE FERMETURE DU VANNAGE À PENTE SIMPLE (COUPLE 3)

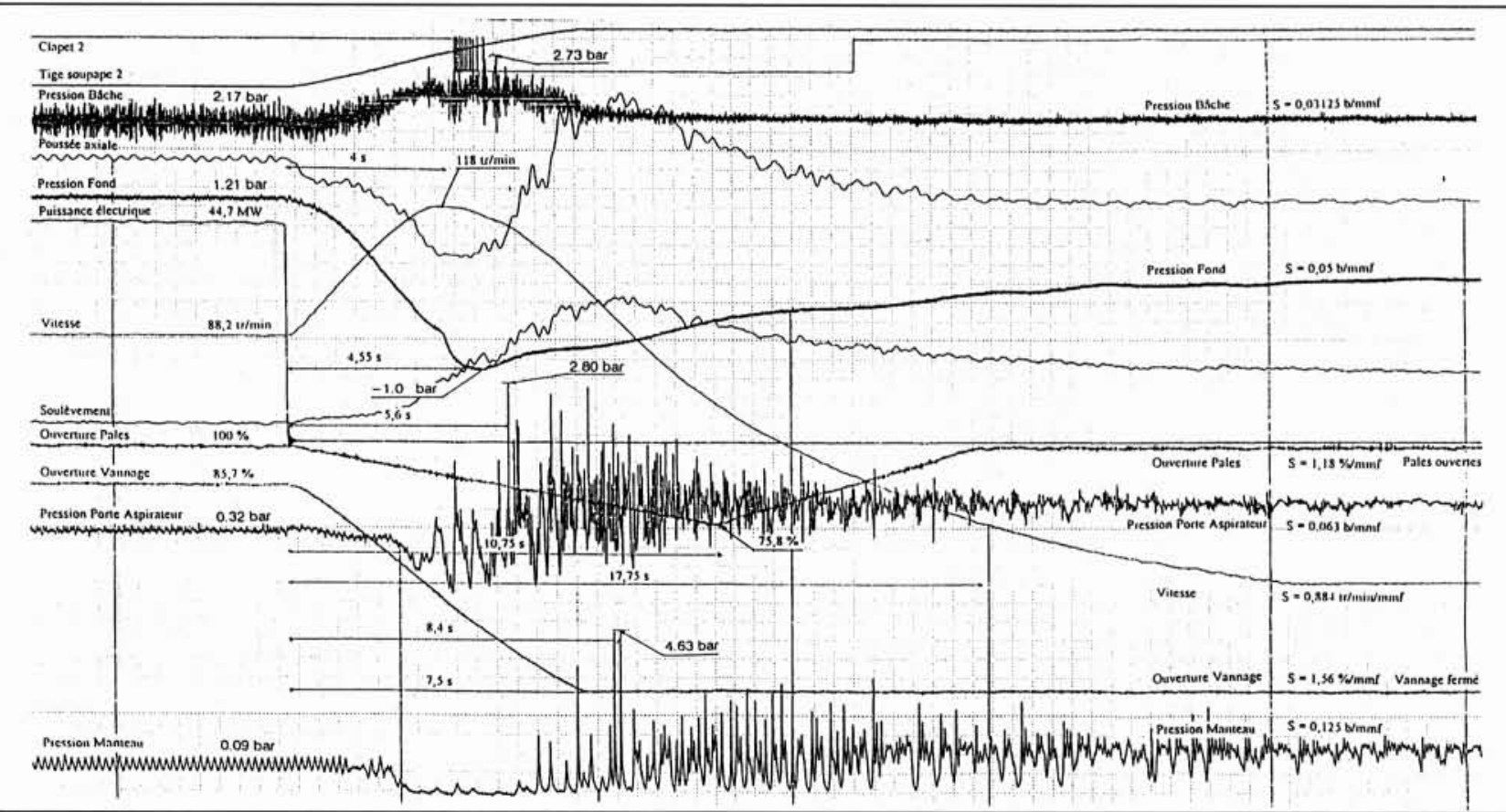


ANNEXE 4 : RESTITUTION TEMPORELLE D'UN ESSAI DE DÉCLENCHEMENT TYPE DB À 45 MW LOI DE FERMETURE DU VANNAGE À PENTE DOUBLE (COUPLE 5).

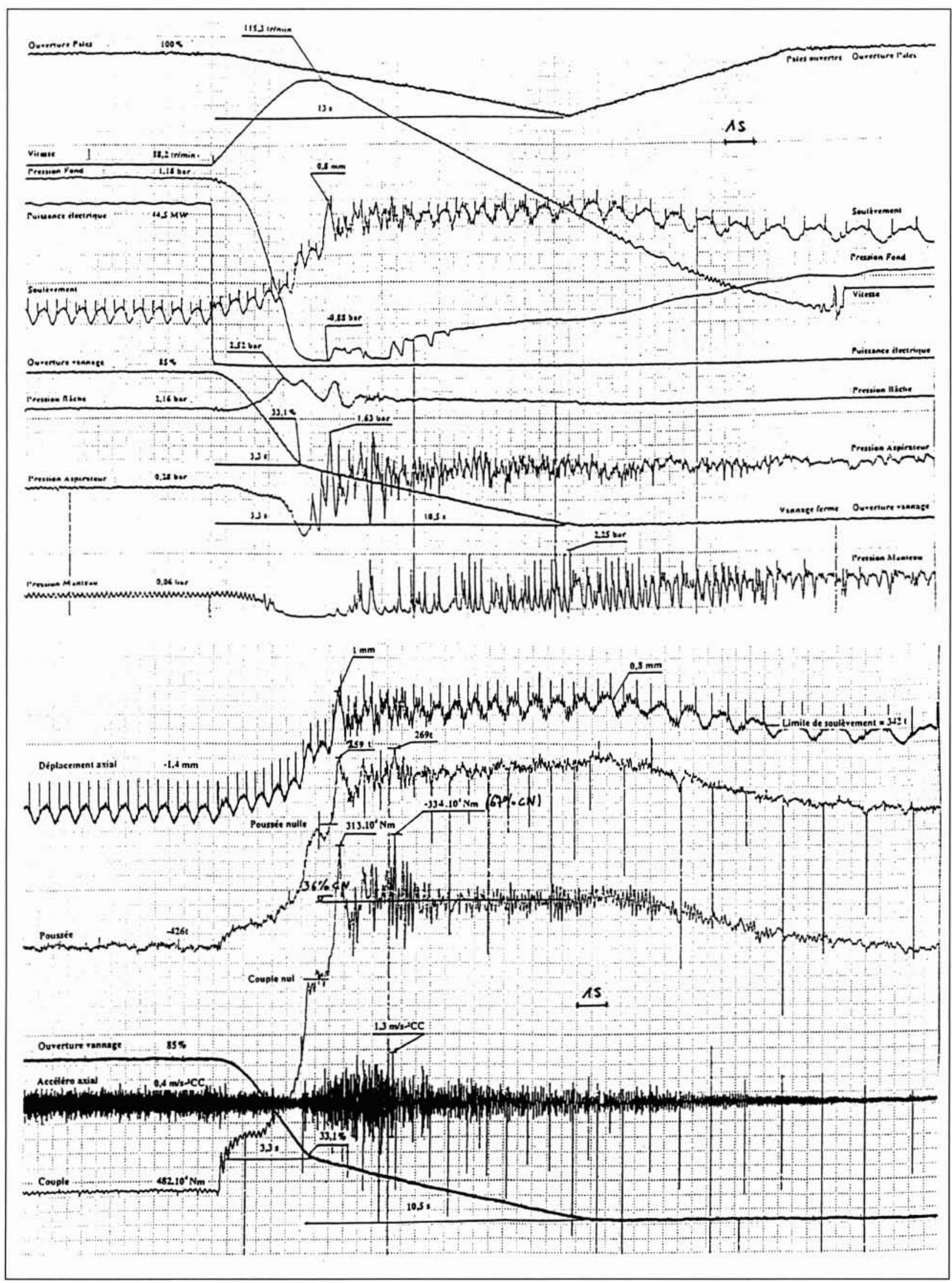




\section{DOSSIER}

ANNEXE 5 : RESTITUTION TEMPORELLE D'UN ESSAI DE DÉCLENCHEMENT TYPE DB À 45 MW LOI DE FERMETURE DU VANNAGE À PENTE DOUBLE (COUPLE 6).

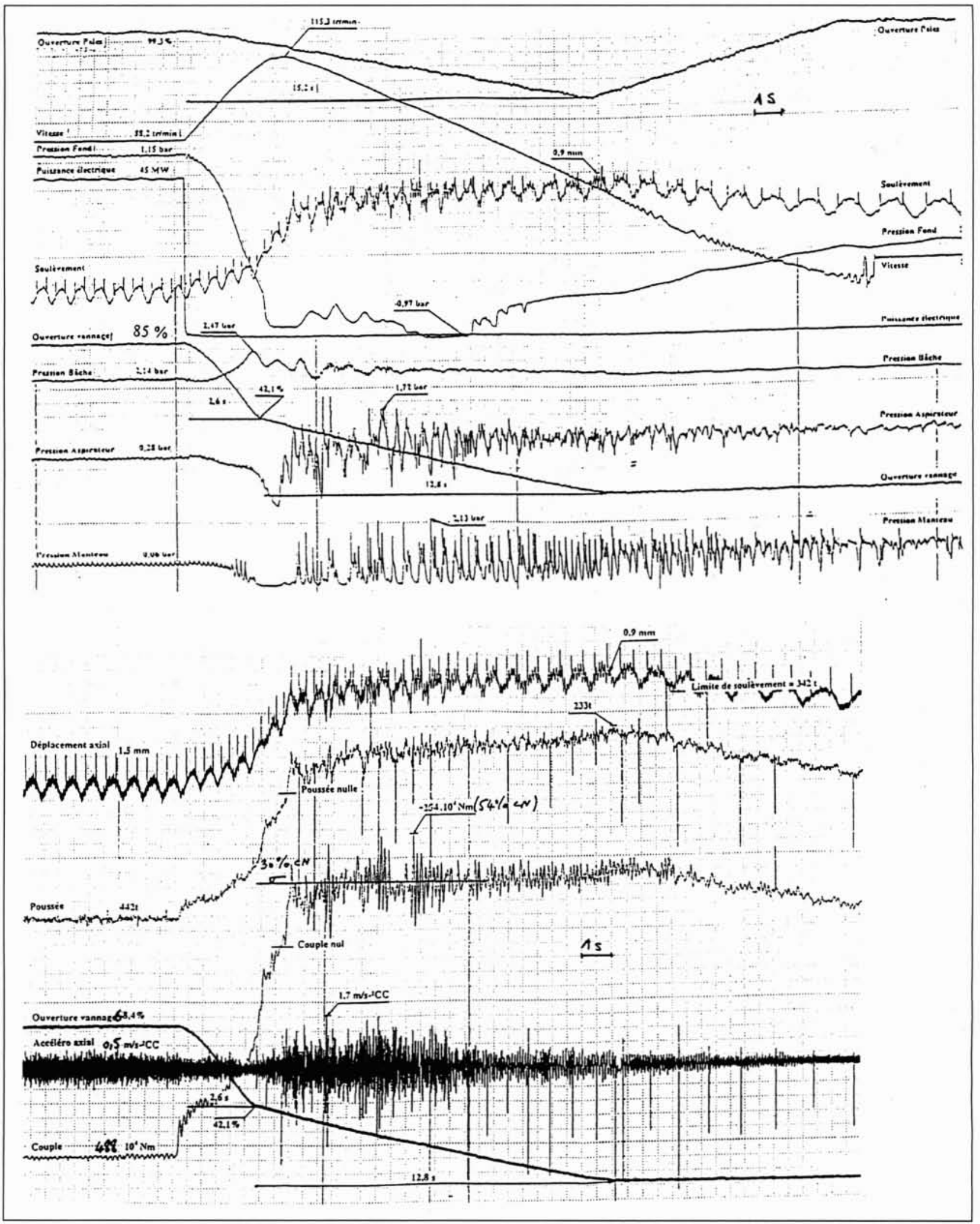

